\title{
Fault Localization in Passive Optical Networks using OTDR Trace Correlation Analysis
}

\author{
Zhixin Liu*, Ming Li and Chun-Kit Chan \\ Department of Information Engineering, The Chinese University of Hong Kong, Shatin, N.T., Hong Kong SAR, China. \\ Tel:+852-2609-8385, Fax: +852-2603-5032,Email:lzx009@ie.cuhk.edu.hk
}

\begin{abstract}
A cost-effective and practical method is proposed for localization of the possible fiber faults in a passive optical network via correlation between the measured OTDR trace and a set of pre-measured reference OTDR traces.

OCIS codes: (060.2330) Fiber optics communications; (120.4825) Optical time domain reflectometry
\end{abstract}

\section{Introduction}

Fiber-to-the-Premises (FTTP) systems implemented by time-division-multiplexed passive optical network (TDMPON) have been deployed worldwide to support high-speed optical broadband access. TDM-PON realizes point-tomultipoint (P2MP) transmission in a tree topology, in which an optical line terminal (OLT) connects to a number of optical network units (ONUs), via a piece of feeder fiber, a $1 \times \mathrm{N}$ power splitter and a set of distribution fibers. In order to assure network availability, physical layer monitoring techniques, especially detection and localization of equipment failure and fiber faults, are indispensable [1]. Traditionally, Optical Time Domain Reflectometry (OTDR) is used to remotely monitor the status of the point-to-point fiber links. However, in P2MP TDM-PONs, the backscattered lights from different branches add together at the remote node (RN) and the OLT, making it difficult to identify the exact faulty fiber branch [2].

Several techniques have been previously proposed to solve this problem. One straightforward approach was to integrate the OTDR function at the ONU, where the embedded OTDR measured the back-reflected and backscattered echoes caused by the upstream light [3]. However, such distributed control and status reporting incurred high complexity in network management. The use of a multi-wavelength OTDR to characterize the individual fiber branches separately was reported in [4], but this solution required costly wavelength-tunable OTDR and other wavelength-specific components at the ONUs. An alternative was to introduce a reference reflection at the end of each fiber branch so as to detect the presence and the height variation of the reference reflection peaks [5]. However, it required distinct fiber length on each branch. Besides, by placing a switchable reflective element (SRE) at each ONU, the fiber branch being monitored can be identified [6]. However, it required dynamic control of all the SREs at the ONU side and extra components have to be installed at each ONU.

In this paper, we propose a practical approach to locate the fiber faults in PONs by calculating the correlations of the measured OTDR trace with a set of hypothesized OTDR traces, which are synthesized by combining the premeasured reference traces for individual fiber branches, under different system conditions. Hence, the faulty fiber branch can be easily identified by selecting the hypothesized OTDR trace with the highest correlation value with the measured OTDR trace at the OLT. This proposed approach does not require any extra component or modification of the system architecture, which makes it a highly compatible and cost-effective solution. We have showed that the proposed solution is also applicable to optical distribution networks that may have fiber branches of equal lengths.

\section{Fiber Fault Localization Methodology}

In a typical TDM-PON, the OTDR pulses launched into the feeder fiber at the OLT are passively power-split at the $\mathrm{RN}$, before being fed simultaneously into all distribution fibers. Based on the fact that different fiber branches in a PON usually give different backscattering/reflection profiles due to their differences in fiber length, attenuation, bending/strain of the fiber cable, as well as the end-reflection peak, and the OTDR trace obtained at the OLT is simply the sum of the back-reflected and back-scattered lights from the feeder fiber and all the fiber branches. In case of a single fiber cut occurred in one of the fiber branches, a sharp step decrement in the received power level will be observed in the aggregated OTDR trace at the OLT. Thus, the relative distance of the fiber cut from the OLT can be estimated. However, it is impossible to identify the faulty fiber branch without any additional information. In our proposed approach, a set of reference OTDR traces for individual fiber branches, under the condition that either all fiber branches, except the one under test, are terminated or they are terminated one at a time, at the RN, are measured at the system initial setup or maintenance period. In the practical scenario with the known relative distance of the fiber cut from the OLT from the measured OTDR trace, a set of hypothesized aggregated OTDR traces can be synthesized from the pre-measured reference OTDR traces, considering all possible faulty fiber branch scenarios in the PON. Hence, by performing correlation analysis between the measured OTDR trace and this set of hypothesized 
aggregated OTDR traces, the one with the highest correlation can be selected and the faulty fiber branch can be determined.

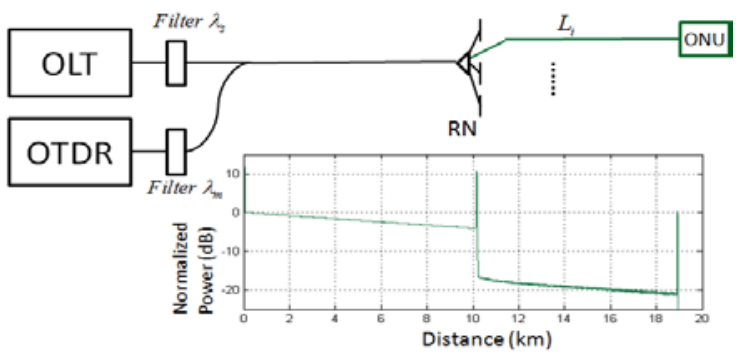

$$
L_{\text {agg_normal }}(d)_{(d B)}= \begin{cases}10 \log _{10}\left[\frac{1}{N} \sum_{i=1}^{N} 10^{\left(\frac{L_{i}(d)}{10}\right)}\right], & d \leq d_{R N} \\ 10 \log _{10}\left[\sum_{i=1}^{N} 10^{\left(\frac{L_{i}(d)}{10}\right)}\right], & d \geq d_{R N}\end{cases}
$$

Fig. 1 System setup for measuring the traces for each branch

Fig. 1 shows the configuration for measuring the reference OTDR traces for each fiber branch. The OTDR operating at a wavelength different from those of the downstream signals is inserted at the OLT. At the RN, all output ports for downstream signals, except the one connected to the fiber branch under measurement, are terminated. Then, the trace for the branch $L_{i}(i=1, \ldots, N)$ is measured, as shown in the inset of Fig. 1, for instance. The measured reference OTDR trace for branch $L_{i}$ is $L_{i_{-} \text {measured }}(d)_{(d B)}$, which is a function of the distance, $d$ (in km), from the OLT. It is then normalized as $L_{i}(d)_{(d B)}=L_{i_{-} \text {measured }}(d)_{(d B)}-L_{i_{-} \text {measured }}\left({ }_{(d B)}\right.$. When the whole system is working normally, the relationship between the aggregated OTDR trace measured at the OLT, $L_{\text {agg_normal }}(d)$, and the measured reference OTDR traces for each branch $L_{i}(d)$, is shown in (1), where $N$ is the number of fiber branches, $d_{R N}$ is the distance (in $\mathrm{km}$ ) from the OLT to the RN. When one single fiber fault occurs, there will be an additional step decrement in the measured aggregated OTDR trace at the OLT, and the distance between the fiber fault and the OLT can be determined, as $d_{\text {fault }}$. Then a set of $N$ hypothesized aggregated OTDR traces can be synthesized from the reference OTDR traces from (1), considering all possible cases of the faulty fiber branch happened at the distance $d_{\text {fault }}$. Finally, correlation analysis between the measured aggregated OTDR trace and all hypothesized aggregated OTDR traces are performed, and the one with the highest correlation is chosen to declare the exact faulty fiber branch.

\section{Experimental Verification}

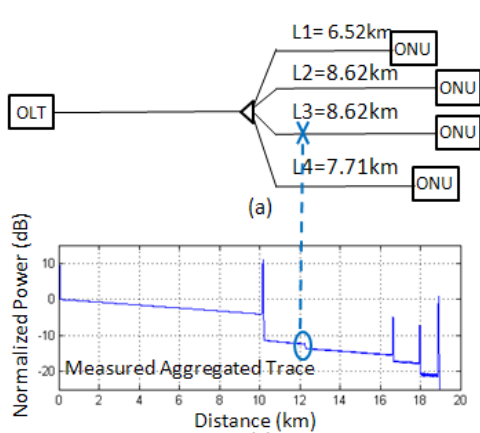

(c)

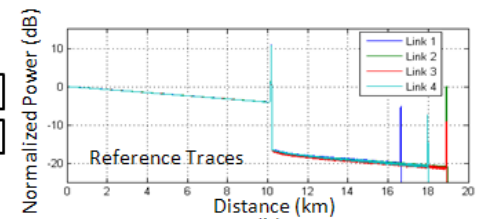

(b)

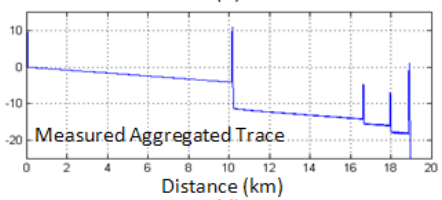

(d)

Fig.2 (a) PON setup in the experiment. (b) Measured reference traces. (c) Measured aggregated trace when fault occurs in L3. (d) Measured aggregated trace when system is normal

The proposed method was verified by a proof-of-concept experiment with a $1 \times 4$ PON setup, as shown in Fig. 2 (a). $d_{R N}$ was $10.2 \mathrm{~km}$ and two of the four fiber branches, L2 and L3, had similar lengths. The OTDR model was Agilent N3910AM and the OTDR pulse width was $300 \mathrm{~ns}$, which corresponded to a distance resolution of about 30 meters. The OTDR was operated at $1625 \mathrm{~nm}$, as this wavelength was more sensitive to fiber bending and pressure, and it did not interfere with the in-service data traffic. The pre-measured reference OTDR traces and the measured aggregated OTDR trace were shown in Fig. 2 (b) and (d), respectively. The reference traces showed different fiber lengths, endreflection peaks, and attenuation values for different fiber branches. In the experiment, the slopes of the reference OTDR traces were close to each other, as the fiber reels used were of similar specifications. In practice, there may be greater differences between the reference traces, as fibers of different specifications may be used in different fiber branches of the deployed PON. The fiber fault considered in the experiment was strong fiber bending and the light in the fiber branch almost completely vanished beyond the fault point. The same algorithm could be utilized for fiber break event. As depicted in Fig. 2 (c), when a single fiber fault in the distribution fiber occurred, a loss event at a 
distance of $d_{\text {fault }}$ from the OLT could be detected on the measured aggregated OTDR trace. Then the hypothesized OTDR traces can be derived by

$$
L_{\text {agg_hyp }}^{m}(d)_{(d B)}=\left\{\begin{array}{l}
L_{\text {agg_normal }}(d), \quad d<d_{\text {fault }} \\
10 \log _{10}\left[10^{\left(\frac{L_{\text {agg_normal }}(d)}{10}\right)}-10^{\left(\frac{L_{m}(d)}{10}\right)}\right], \quad d>d_{\text {fault }}
\end{array} \quad m=1,2,3,4\right.
$$

where $m$ was the index for the fiber branch that was faulty in the hypotheses. The measured aggregated trace and the calculated hypothesized aggregated traces were shown in the upper diagrams in Figs. 3 (a)-(d), in which L1, L2, L3, and L4 were supposed to be faulty, respectively. The lower diagrams in Figs. 3 (a)-(d) showed differences between the hypothesized aggregated traces and the measured aggregated trace. The calculated correlation coefficients between the calculated hypothesized aggregated traces and the measured aggregated trace, which were based on the mean square error of the trace difference, were $0.006,0.026,0.710$, and 0.007 , respectively. The highest correlation was observed when L3 was supposed to be faulty. Therefore, the faulty fiber L3 was successfully identified.

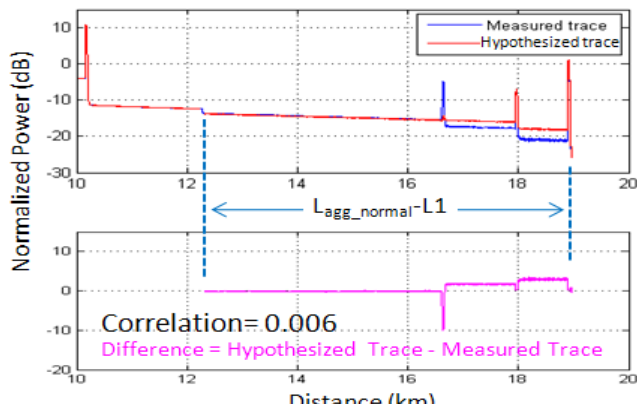

(a)

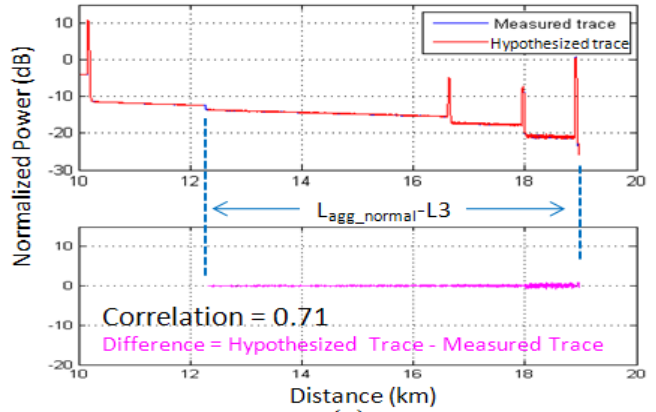

(c)

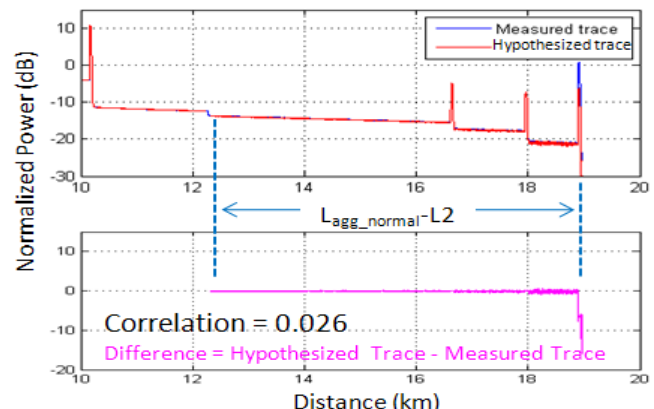

(b)

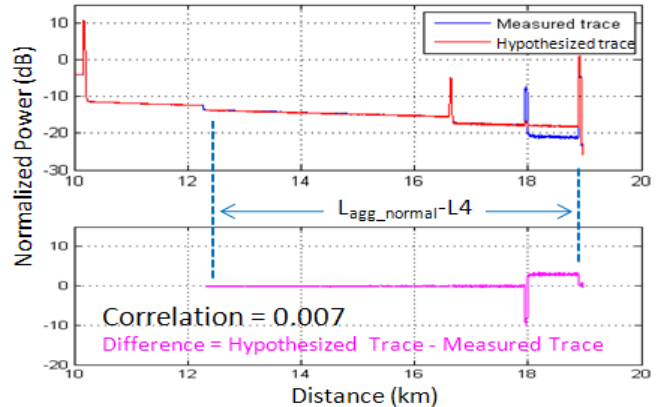

(d)

Fig. 3 Measured aggregated OTDR traces \& hypothesized aggregated OTDR traces (upper graphs), and their differences (bottom graphs), when the hypothesized faulty branch is (a) L1, (b) L2, (c) L3, and (d) L4, respectively.

\section{Summary}

We have proposed a novel fault localization method in TDM-PON by comparing the correlation coefficients between the measured aggregated OTDR trace and a set of hypothesized aggregated OTDR traces synthesized by a set of pre-measured OTDR traces of individual fiber branches. The feasibility of this method has been experimentally verified in a $1 \times 4 \mathrm{PON}$. The proposed approach is still valid when the PON has branches of equal lengths and it enables a cost-effective and reliable means in locating fiber faults in PONs.

\section{References}

[1] F. Caviglia, V. C. Di Biase, A. Gbazzi, "Optical maintenance in PONs”, European Conf. on Optical Commun., Madrid, 1998, pp. 621-626.

[2] K. Yuksel, V. Moeyaert, M. Wuilpart, and P. Mégret, "Optical layer monitoring in passive optical networks (PONs): A review", in Proc. ICTON 2008, pp. 92-98, paper Tu. B1.1, 2008.

[3] H. Schmuck, J. Hehmann, M. Straub and Th. Pfeiffer, "Embedded OTDR techniques for cost-efficient fibre monitoring in optical access networks", Proc. of the 32th European Conference on Optical Communications, ECOC'06, Cannes, Mo3.5.4, 2006.

[4] K. Ozawa, M. Shigehara, J. Hanai, A. Ban, T. Naitou, K. Shimoura, "Field trial of in-service individual line monitoring of PONs using a tunable OTDR", Proc. of the 14th Conference on Optical Fiber Sensors, OFS'00, Venice, pp. 880-883 (2000).

[5] L. Wuilmart, V. Moeyaert, D. Daniaux, P. Megret, and M. Blondel, "A PC-based method for the localisation and quantization of faults in passive tree-structured optical networks using the OTDR technique," in Proc. Symposium on Optical Fiber Measurements, Boulder, CO, October 1-3, 1996.

[6] K. Yuksel, S. Dupont, D. Hamoir and J.-C. Froidure, "FTTx automated test solution: requirements and experimental implementation", Electro. Lett., vol. 41, no. 9, pp. 546-547, (2005). 